\title{
Ultrasound-targeted microbubble destruction-mediated downregulation of CD133 inhibits epithelial-mesenchymal transition, stemness and migratory ability of liver cancer stem cells
}

\author{
YAN-MIN LIU ${ }^{1}$, XUAN-FEI LI ${ }^{2}$, HAO LIU ${ }^{3}$ and XIAO-LING WU ${ }^{1}$ \\ ${ }^{1}$ Department of Gastroenterology and Hepatology and ${ }^{2}$ Department of Hepatobiliary Surgery, The Second Affiliated Hospital \\ of Chongqing Medical University, Chongqing 400010; ${ }^{3}$ Department of Gastroenterology, University-Town Hospital \\ of Chongqing Medical University, Chongqing 401331, P.R. China
}

Received June 25, 2015; Accepted July 29, 2015

DOI: 10.3892/or.2015.4270

\begin{abstract}
Hepatocellular carcinoma (HCC) is an aggressive disease with a poor outcome due to the high incidence of metastasis. Cancer stem cells (CSCs) have been identified to be responsible for tumor progression and may be generated by epithelial-mesenchymal transition (EMT) characteristics. CD133 is a specific surface marker for liver cancer stem cells (LCSCs), which is also considered as an important functional factor for tumorigenesis and overall survival in HCC. Ultrasound-targeted microbubble destruction (UTMD) has recently been used as a novel, safe and effective gene transfection technology. The aim of the present study was to elucidate the regulatory mechanism of CD133 and EMT in LCSCs and whether the UTMD-based shRNA delivery system facilitated gene delivery in LCSCs. In the present study, CD133 ${ }^{+}$cells were isolated from the SMMC-7721 HCC cell line and then transfected with shCD133 mediated by UTMD and liposomes, respectively. Compared to the liposomes group, the UTMD group resulted in significantly improved transfection efficiency. The downregulation of CD133 reversed the EMT program, attenuated self-renewal, proliferation and migration of CD133+ LCSCs and suppressed the growth of CSC tumor xenografts. Additionally, the downregulation of CD133 led to downregulation of the nuclear factor- $\kappa \mathrm{B}(\mathrm{NF}-\kappa \mathrm{B})$ pathway.
\end{abstract}

Correspondence to: Professor Xiao-Ling Wu, Department of Gastroenterology and Hepatology, The Second Affiliated Hospital of Chongqing Medical University, 74 Linjiang Road, Chongqing 400010, P.R. China

E-mail: wxlcqmu1@163.com

Dr Hao Liu, Department of Gastroenterology, University-Town Hospital of Chongqing Medical University, Chongqing 401331, P.R. China

E-mail: 1hcqmu@163.com

Key words: hepatocellular carcinoma, cancer stem cells, CD133, epithelial-mesenchymal transition, ultrasound-targeted microbubble destruction, NF- $\kappa \mathrm{B}$
The present study demonstrated that CD133 plays a critical role in the regulation of the EMT process, tumor-initiating properties and migratory ability of LCSCs. The UTMD technique targeted for CD133 downregulation may be examined as a potential therapeutic strategy for HCC.

\section{Introduction}

Hepatocellular carcinoma (HCC) is the sixth most prevalent cancer worldwide and the third most frequent cause of cancer-related mortality (1). Surgical resection and liver transplantation are currently the main options for the treatment of HCC, however, the high frequency of tumor recurrence is almost inevitable due to therapy resistance (2). Accumulating scientific evidence indicates that tumor formation is driven by a subpopulation of self-renewing cells known as cancer stem cells (CSCs) or tumor-initiating cells (TICs) (3). CSCs have been shown to be responsible for tumor initiation, metastasis, recurrence and chemoresistance (4). CSCs in liver cancer cells can be identified and enriched by several specific surface markers, such as CD90, CD44 and most recently, CD133 (5). CD133 (also known as AC133 or prominin-1), a 5 transmembrane cell surface glycoprotein, has been used to extract a subset of putative stem cells in HCC. Moreover, CD133+ liver cancer cells possess many stem cell properties, including self-renewal, high proliferation, differentiation and have greater tumorigenicity and chemoresistance (6-8). As a liver CSC marker, CD133 also serves as an important indicator for tumor malignant progression, patient survival and recurrence rates $(9,10)$.

The epithelial-mesenchymal transition (EMT) is a transdifferentiation process that converts adherent epithelial cells into migratory mesenchymal cells. Activation of the EMT program is considered an important step in the embryonic development, tumorigenic progression and cancer metastasis (11). Previous studies have also linked EMT with the properties of CSCs in HCC (12). Several associated signalling pathways are able to induce EMT, such as nuclear factor- $\kappa \mathrm{B}$ (NF-kB), Notch, TGF- $\beta$ and Wnt $/ \beta$-catenin, which are also known to regulate 
CSCs (13). As a transcription factor, $N F-\kappa B$ has been reported to play critical roles in the processes of EMT, tumor invasion and metastasis and the maintenance of CSCs in various types of cancer, including liver cancer (14). Activation of NF- $\kappa \mathrm{B}$ typically involves the phosphorylation of the inhibitor of $\kappa \mathrm{B}-\alpha$ $(\mathrm{I} \kappa \mathrm{B} \alpha$ ) by the I $\kappa \mathrm{B}$ kinase (IKK) complex. Moreover, NF- $\kappa \mathrm{B}$ activation through IKK activity modulation leads to EMT marker changes and the development of CSCs (15).

With the emergence of new biotechnologies in gene delivery, ultrasound-targeted microbubble destruction (UTMD) has evolved into a new, safe, non-viral gene transfection tool for site-specific drug and gene delivery $(16,17)$. It has been shown that ultrasound microbubble-mediated delivery enhances the efficacy of gene transfection and reduces the side-effects of other bioactive transfection agents, such as liposomes and viral vectors. Recombinant expression plasmid of the shRNA targeting gene mediated by the UTMD technique specifically and effectively regulated the expression of target genes in several studies $(18,19)$. Based on those studies, we hypothesized that combining UTMD and shRNA is a promising strategy for gene delivery in liver cancer stem cells (LCSCs).

Mounting evidence suggests that the stem cell-related CD133 gene and the EMT process have a linear relationship. However, few studies have shown this association in LCSCs. In the present study, using the UTMD technique, we demonstrated that CD133 plays a vital functional role in the regulation of EMT, tumor-initiating properties and migratory ability of LCSCs in vitro and in vivo. Additionally, we found that the reversal of EMT and the impaired invasion and migration of LCSCs by CD133 downregulation may be in part, associated with the repression of the NF- $\mathrm{B}$ pathway. Findings from the present study provide insight into the regulatory effects of CD133 on EMT transformation in LCSCs and lead to the development of new and more effective therapeutics for HCC.

\section{Materials and methods}

Cell lines and culture. The human SMMC-7721 liver cancer cell line was obtained from the Institute of Life Sciences of Chongqing Medical University (Chongqing, China). SMMC-7721 cells were maintained in Dulbecco's modified Eagle's medium (DMEM) supplemented with $10 \%$ fetal bovine serum (FBS) (both from Gibco, Life Technologies, Carlsbad, CA, USA), $100 \mathrm{U} / \mathrm{ml}$ penicillin and $100 \mu \mathrm{g} / \mathrm{ml}$ streptomycin (Invitrogen Life Technologies, Carlsbad, CA, USA). CD133+ cells sorted from the SMMC-7721 cells were performed in serum-free culture medium (SFM) composed of DMEM/F12 (Gibco) plus $20 \mathrm{ng} / \mathrm{ml}$ basic fibroblast growth factor (bFGF), $20 \mathrm{ng} / \mathrm{ml}$ epidermal growth factor (EGF) (both from PeproTech, Rocky Hill, NJ, USA) and 2\% B27 supplement (Life Technologies). The cells were cultured in a humidified incubator with $5 \% \mathrm{CO}_{2}$ at $37^{\circ} \mathrm{C}$.

Flow cytometric analysis and fluorescence-activated cell sorting (FACS). SMMC-7721 cells were subjected to FACS. The cells were dissociated, washed and resuspended in phosphate-buffered saline (PBS). Human-specific anti-CD133/1 (AC133) conjugated to R-phycoerythrin (PE; Milyteni Biotec, Bergisch Gladbach, Germany) and anti-CD133-florescein isothiocyanate mouse monoclonal antibody were used for
FACS analysis. All the procedures were performed according to the manufacturer's instructions. The labeled cells were analyzed and sorted by the FACS-LSR II flow cytometer (Becton-Dickinson, Franklin Lakes, NJ, USA). The fresh isolated CD133+ ${ }^{+}$SMMC-7721 cells were collected and cultured in the SFM.

Experimental grouping. To analyze the effect of UTMD and liposomes on CD133 transfection and the biological characteristics of LCSCs, the fresh sorted CD133+ SMMC-7721 cells were divided into the following three groups: the control (control); shCD133 plasmid + Lipofectamine $2000(\mathrm{P}+\mathrm{L})$; and the shCD133 plasmid + ultrasound exposure + microbubbles group (P+UTMD).

Lipofectamine-mediated gene transfection and ultrasoundtargeted microbubble destruction (UTMD) exposure protocols. The gene-specific short hairpin RNA (shRNA) (HuSH 29-mer shRNA constructs against PROM1 in pGFP-VRS vector) were obtained from OriGene (Rockville, MD, USA). The cells in the $\mathrm{P}+\mathrm{L}$ group were transfected with shCD133 and Lipofectamine 2000 protocol (Invitrogen Life Technologies) in Opti-MEM medium without serum with a ratio of 1:2 according to the manufacturer's instructions. The mixture was incubated for $5 \mathrm{~min}$ at room temperature prior to being added to the cells. A therapeutic ultrasound machine (Institute of Ultrasound Imaging, Chongqing Medical University) was used and the area of the probe $(1 \mathrm{MHz})$ was $\sim 5 \mathrm{~cm}^{2}$. Microbubbles (SonoVue; Bracco, Milan, Italy) were lipid-shelled ultrasound contrast agents containing sulfur hexafluoride gas (diameter, 1.0-10.0 $\mu \mathrm{m}$ ) and used at a concentration of $\sim 2 \times 10^{8}$ bubbles $/ \mathrm{ml}$. SonoVue was reconstituted in saline solution according to the manufacturer's instructions prior to transfection. Plasmid DNA and SonoVue complexes were gradually added to cell suspensions in the P+UTMD group. After protocol optimization with various settings, the UTMD parameters were set at the radiation frequency of $1 \mathrm{MHz}, 20 \%$ duty cycle and sound intensity of $1 \mathrm{~W} / \mathrm{cm}^{2}$ for $60 \mathrm{sec}$. The plates were supplemented with $2 \mathrm{ml}$ serum-free culture medium and incubated in an incubator at $37^{\circ} \mathrm{C}$ and $5 \% \mathrm{CO}_{2}$ until gene expression analysis was performed. The shRNA expression vectors expressed green fluorescent protein (GFP) that was used to track transfection efficiency. At 12, 24 and $48 \mathrm{~h}$ after transfection, transfection efficiency was evaluated by observing the expression of GFP in the living cells with fluorescent microscopy (Olympus, Tokyo, Japan). After $48 \mathrm{~h}, \mathrm{RT}-\mathrm{qPCR}$ and western blotting were used to determine the levels of related gene expression.

Reverse transcriptase-quantitative PCR analysis. Total RNA from cells was isolated using the TRIzol reagent (Takara, Dalian, China) according to the manufacturer's instructions. The concentrations and purity of the total RNA were evaluated using a UV spectrophotometer(Ultrospec 2100 Pro; Amersham, USA). Total RNA was reverse transcribed into cDNA using the PrimeScript RT reagent kit (Takara). Quantitative PCR (RT-qPCR) was performed using SYBR Premix Ex Taq $^{\mathrm{TM}}$ II (Takara) with the CFX96 ${ }^{\mathrm{TM}}$ Real-Time System (Bio-Rad, Hercules, CA, USA). GAPDH was used as an internal control. The relative expression levels of mRNAs were calculated using 
the $2^{-\Delta \Delta \mathrm{Ct}}$ method. RT-qPCR reactions were run in triplicate. Primer sequences for the genes analyzed were: CD133 forward, 5'-AGG ACA AGG CGT TCA CAG AT-3' and reverse, 5'-ACC AAG CAC AGA GGG TCA TT-3'; CD44 forward, 5'-AAG GAG CAG CAC TTC AGG AG-3' and reverse, 5'-ATC CCA GGT TTC TTG CCT CT-3'; CD90 forward, 5'-GAC CCG TGA GAC AAA GAA GC-3' and reverse, 5'-GCC CTC ACA CTT GAC CAG TT-3'; Oct4 forward, 5'-ACA TGT GTA AGC TGC GGC C-3' and reverse, 5'-GTT GTG CAT AGT CGC TGC TTG-3'; Sox2 forward, 5'-TCC CGT ATG AAA GCA TCG TGG-3' and reverse, 5'-CCC ATT TGG GTA GAT CAG GTA AC-3'; E-cadherin forward, 5'-GGA TGT GCT GGA TGT GAA TG-3' and reverse, 5'-TG GGC AGT GTA GGA TGT GAT-3'; N-cadherin forward, 5'-CGT GAA GGT TTG CCA GTG T-3' and reverse, 5'-CAG CAC AAG GAT AAG CAG GA-3'; vimentin forward, 5'-AGA GAA CTT TGC CGT TGA AGC-3' and reverse, 5'-ACG AAG GTG ACG AGC CAT T-3'; GAPDH forward, 5'-AGA AGG CTG GGG CTC ATT TG-3' and reverse, 5'-AGG GGC CAT CCA CAG TCT TC-3'.

Cell sphere formation and colony formation assays. For sphere formation assays, single CD133+ SMMC-7721 cells of the three groups were plated in a 6-well ultra-low attachment plate at a density of $1 \times 10^{3}$ cells/ml (Corning, Steuben, NY, USA) in the SFM. Sphere formation (diameter, $>50 \mu \mathrm{m}$ ) in each well was calculated under an inverted microscope (Olympus) on the 14th day after seeding.

Colony formation assays were carried out two days after transfection. Cells (500/group) were resuspended in DMEM with $10 \%$ FBS and were plated in the 6-well plates. The culture medium was changed twice/week. After 14 days of incubation, the cells were fixed in $4 \%$ formaldehyde and stained with Giemsa staining (Santa Cruz Biotechnology, Inc., Santa Cruz, CA, USA). Colonies with $>50$ cells were counted.

Cell proliferation assay and apoptosis detection. Cell proliferation was evaluated by the Cell Counting Kit-8 (CCK-8) (Dojindo, Kumamoto, Japan) assays. The cells were plated at a density of $2 \times 10^{3}$ cells/well in 96-well plates containing serum-free culture medium. Cell proliferation was examined after 1, 2, 3, 4 and 5 days, respectively. CCK-8 reagent $(10 \mu \mathrm{l})$ was added to each well and incubated for $4 \mathrm{~h}$ at $37^{\circ} \mathrm{C}$. The spectrophotometric absorbance at 450-nm wavelength was recorded using a microplate reader (Bio-Rad).

Flow cytometry was used to investigate cell apoptosis. The target cells were collected and resuspended in PBS buffer at a concentration of $1 \times 10^{6}$ cells/tube. The apoptotic rate of cells was detected with an Annexin V-FITC apoptosis detection kit (eBioscience, Inc., San Diego, CA, USA) and propidium iodide (PI; Sigma-Aldrich) double staining according to the manufacturer's guidelines. Flow cytometric analysis was performed by a FACS-LSR II flow cytometer.

Cell migration and invasion assays. For the Transwell migration assays, $1 \times 10^{5}$ cells of the three groups were, respectively, resuspended in $200 \mu \mathrm{l}$ of serum-free culture medium and plated in the upper chamber of a $24-w e l l ~ 8-\mu \mathrm{m}$ pore size Transwell plate (Corning, New York, NY, USA). The lower chamber of Transwell was loaded with $600 \mu$ l culture medium supplemented with $10 \%$ FBS as a chemoattractant. The plates were incubated at $37^{\circ} \mathrm{C}$ in $5 \% \mathrm{CO}_{2}$ for $24 \mathrm{~h}$. Non-migrating cells on the upper chamber were removed from the surface of the membrane with cotton-tipped swabs. The cells migrating through the lower membrane were fixed with $4 \%$ paraformaldehyde for $30 \mathrm{~min}$ and stained with $0.1 \%$ crystal violet for $15 \mathrm{~min}$. The cells were counted under a light microscope (Olympus) at a magnification of x200 in five randomized fields. Transwell invasion assay was similarly performed through the Boyden chamber with polycarbonate membrane inserts that were coated with BD Matrigel Basement Membrane Matrix (BD Biosciences, San Jose, CA, USA).

Western blot analysis. Protein was extracted from the cells using RIPA lysis buffer (Beyotime, Shanghai, China) with protease and phosphatase inhibitors (Thermo Scientific, Waltham, MA, USA). The protein concentration was determined using a BCA kit (Beyotime, Nanjing, China). Equal amounts of protein cell lysates were separated on SDS-PAGE gels and transferred to PVDF membranes (Millipore, Billerica, MA, USA). The membranes were blocked in 5\% non-fat dry milk in Tris-buffered saline with $0.1 \%$ Tween-20 (TBST) for $1 \mathrm{~h}$ at room temperature and incubated with primary antibodies overnight at $4^{\circ} \mathrm{C}$. The primary antibody dilutions used were: anti-E-cadherin, anti-N-cadherin and anti-vimentin (Abcam, Cambridge, UK) at a dilution of 1:1,000; anti- $\beta$-actin (Santa Cruz Biotechnology, Inc.), 1:2,000; anti-IKK $\beta$, anti-p-IKK $\beta$, anti-I $\kappa \mathrm{B} \alpha$, anti-p-I $\kappa \mathrm{B} \alpha$, anti-RelA and anti-p-RelA (Cell Signaling Technology, Inc., Danvers, MA, USA) at a dilution of 1:1,000. The membranes were washed with TBST and then incubated with the secondary antibodies for $1 \mathrm{~h}$. The protein bands were then visualized using an enhanced chemiluminescence (ECL) reagent (KeyGen, Nanjing, China) and quantified using the ChemiDoc ${ }^{\mathrm{TM}}$ XRS detection system (Bio-Rad).

Xenograft tumorigenicity assay. Four-week-old male BALB/c nude mice were purchased from the Laboratory Animal Center of Chongqing Medical University (Chongqing, China) to examine tumorigenicity. The mice were raised in a specific pathogen-free unit under isothermal conditions. To evaluate the role of CD133 in tumor formation, three groups of cells $\left(5 \times 10^{5}\right)$ were suspended in $100 \mu \mathrm{l}$ of serum-free medium and inoculated subcutaneously into the flanks of nude mice. Tumor diameters were measured every week using vernier calipers. After 5 weeks, the mice were euthanized, and tumors were weighed. Tumor volumes were calculated according to the formula: $\mathrm{v}\left(\mathrm{mm}^{3}\right)=$ length $\mathrm{x}$ width ${ }^{2} / 2$. The tissue samples were preserved for subsequent experiments. All the experimental protocols were approved by the Animal Ethics Committee of Chongqing Medical University. All the procedures involving animals were conducted as indicated by the guidelines of the National Institutes of Health Guide for the Care and Use of Laboratory Animals.

Immunohistochemistry. Briefly, tissue samples preserved in formalin were embedded in paraffin blocks and sectioned into positively charged microscope slides. The sections were deparaffinized in xylene and rehydrated in graded alcohols, and antigens were retrieved by heating at $95^{\circ} \mathrm{C}$ for $15 \mathrm{~min}$. The slides were incubated in $3 \%$ hydrogen peroxide for $20 \mathrm{~min}$, and then incubated at $4^{\circ} \mathrm{C}$ overnight with primary 

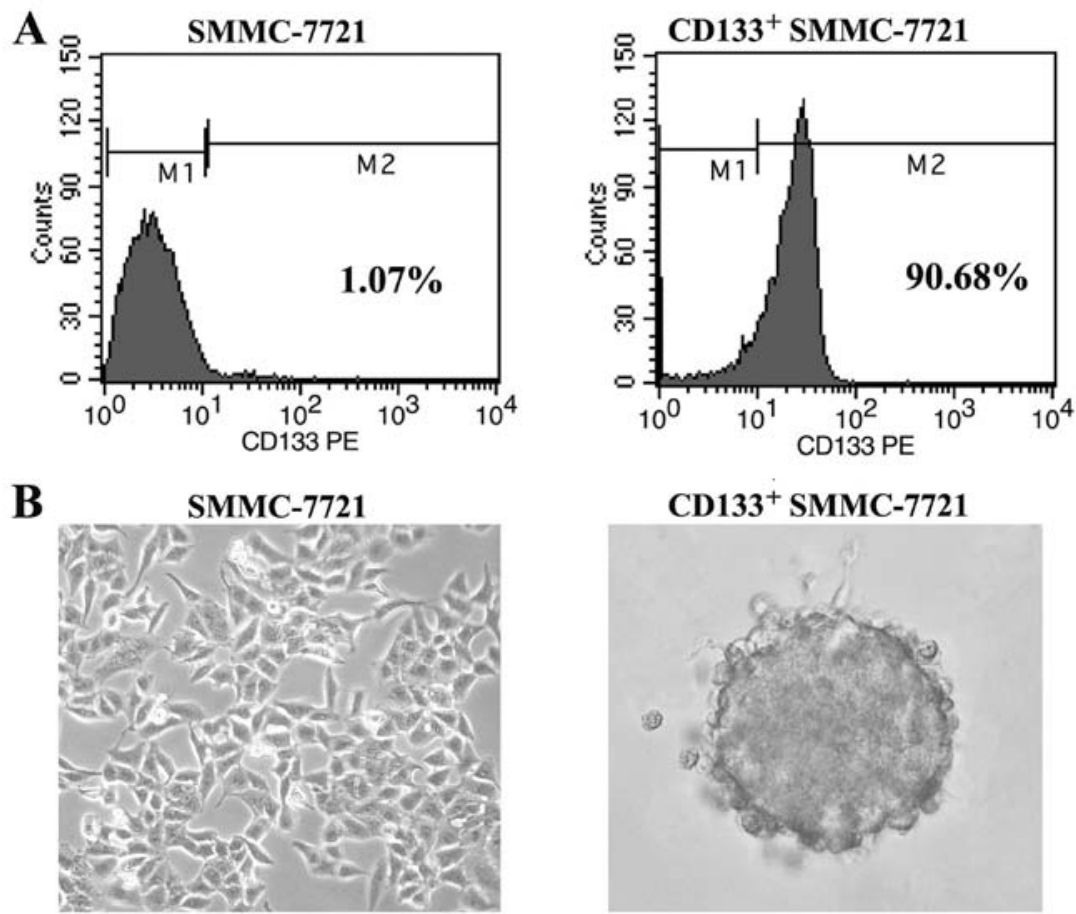

Figure 1. CD133 expression and sorting purity analysis in the SMMC-7721 cell line. (A) Representative phase-contrast micrographs of SMMC-7721 and $\mathrm{CD}_{133^{+}}$cells sorted from SMMC-7721 cells (magnification, $\mathrm{x} 200$ ). (B) Flow cytometry was used to analyze the expression of CD133 in SMMC-7721 cells and FACS-sorted CD133+ SMMC-7721 cells. The rates of CD133 ${ }^{+}$cells in SMMC-7721 cells were $0.2-2 \%$. FACS sorted CD133 cells exhibited a purity ranging from 80.2 to $90.6 \%$. FACS, fluorescence-activated cell sorting.

antibodies anti-E-cadherin, anti-N-cadherin and anti-vimentin (1:100 dilution; Abcam), respectively. Following incubation with appropriate secondary antibodies for $30 \mathrm{~min}$ at $37^{\circ} \mathrm{C}$, the sections were exposed to streptavidin-HRP label (Zhongshan Chemical, Beijing, China) for $30 \mathrm{~min}$ at $37^{\circ} \mathrm{C}$ and incubated for $15 \mathrm{~min}$ with the chromogen 3,3'-diaminobenzidine and counterstained with hematoxylin. The sections were then dehydrated and mounted with neutral gum (Bioworld Technology, Inc., St. Louis Park, MN, USA). The slides were then observed under a light microscope.

Statistical analysis. Experiments were performed in triplicate. Statistically significant values were determined using SPSS 17.0 software (SPSS, Inc., Chicago, IL, USA). The data are presented as the means \pm standard deviation (SD). Group comparisons were evaluated with the Student's t-test or analysis of variance (ANOVA). $\mathrm{P}<0.05$ was considered to indicate statistically significant differences.

\section{Results}

Isolation of $\mathrm{CD} 133^{+}$cells from the SMMC-7721 HCC cell line. It has been reported that the liver CSC marker CD133 is represented only in a small subset of the tumor population in liver cancer cells (5). In an attempt to characterize the molecular mechanisms by which $\mathrm{CD}_{133^{+}}$liver CSCs mediate tumor formation and growth, we sorted out the CD133+ liver CSCs from the SMMC-7721 HCC cell line by FACS. Following sorting, CD133+ SMMC-7721 cells were analyzed by flow cytometry, resulting in a considerable enrichment in the $\mathrm{CD}_{133}{ }^{+}$cell population (purity, $>90 \%$ ) compared to the unsorted SMMC-7721 cells (purity, 0.2-2\%) (Fig. 1A). To establish a long-term culture from CD133+ ${ }^{+}$SMC-7721 cells that possess cancer stem cell-like properties, we performed the tumorsphere formation assay by culturing the $\mathrm{CD}_{133^{+}}$cells in serum-free culture medium. Within 14 days of culture, we obtained liver cancer spheroids in $\mathrm{CD}_{133^{+}}$cells which grew in aggregate clusters and increased in size and amount in a timely manner (Fig. 1B).

Downregulation of CD133 reduces stemness properties in LCSCs. Since CD133 is a known liver CSC marker, we examined whether the downregulation of CD133 had any effect on the inhibition of cancer and stem cell-like properties, CD133+ SMMC-7721 cells were transfected with shCD133 mediated by UTMD and Lipofectamine 2000, respectively. The effects of the transfection were assessed with fluorescence, RT-qPCR and western blot analysis. We observed the expression of GFP under an inverse fluorescence microscope $12 \mathrm{~h}$ after transfection (Fig. 2A). Forty-eight hours after the transfection, the expression of GFP was the strongest. The expression of GFP was attenuated gradually $72 \mathrm{~h}$ after transfection. The CD133 expression levels of the groups were determined by RT-qPCR and western blot analysis. We observed that the relative mRNA and protein levels of CD133 in the UTMD group of cells were significantly decreased compared to the Lipofectamine or control group cells (Fig. 2B). Furthermore, the mRNAs of several stem cell associated genes, including CD44, CD90, Oct4 and SOX2, were all downregulated in the CD133 knockdown LCSCs, which was confirmed by RT-qPCR (Fig. 2C). We also examined the effect of the downregulation of CD133 on self-renewal 
A

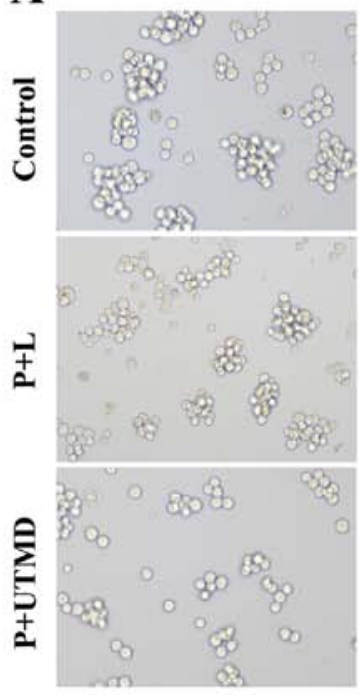

B
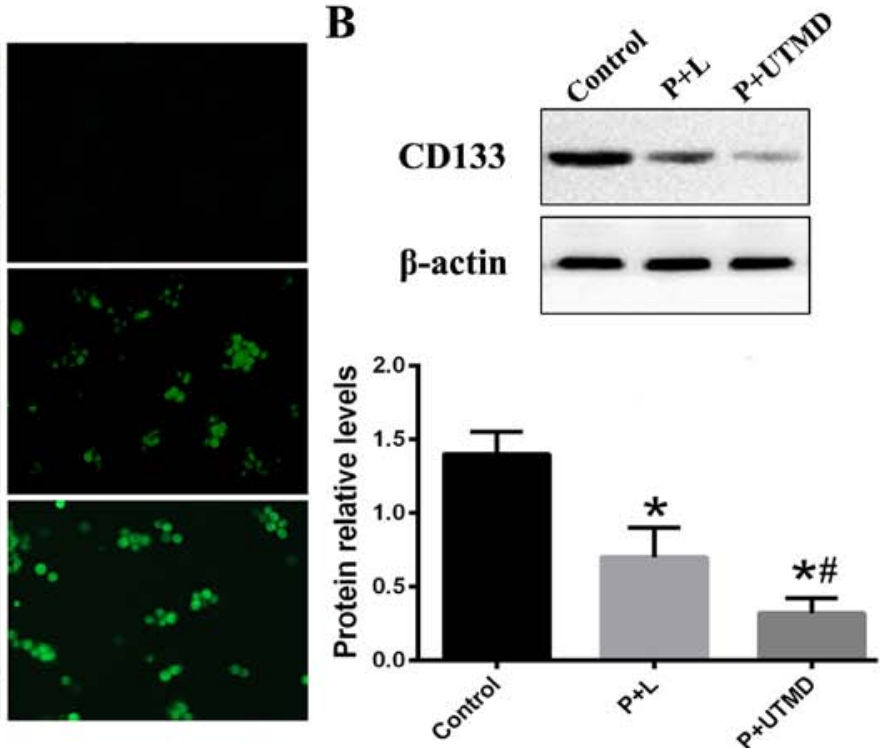

C

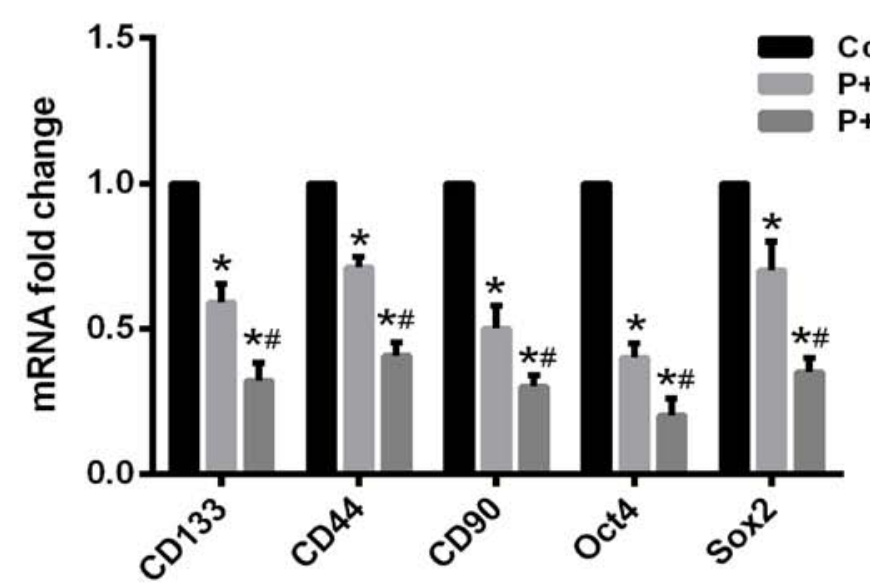

D

Control

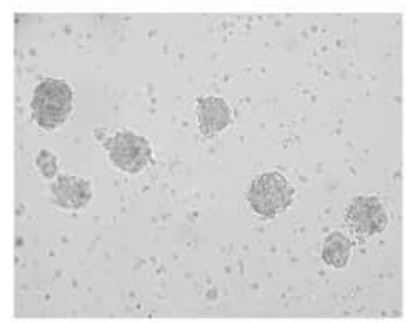

$\mathbf{P}+\mathbf{L}$

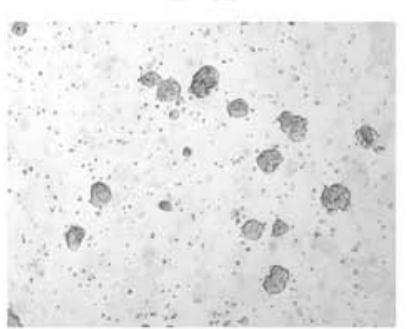

P+UTMD

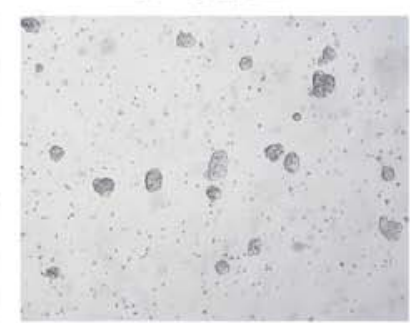

Figure 2. Downregulation of CD133 inhibits self-renewal and sphere-forming ability of CD133+ SMMC-7721 cells. (A) Fluorescent imaging of CD133+ SMMC-7721 cells transfected with shCD133 mediated by ultrasound-targeted microbubble destruction (P+UTMD) or Lipofectamine (P+L) after 48 h (magnification, x100). (B and C) The transfection efficiency of shCD133 in CD133+ SMMC-7721 cells was validated by RT-qPCR and western blot analysis. The relative mRNA levels of CD44, CD90, Oct4 and Sox 2 were detected by RT-qPCR assay. $\beta$-actin was used as an internal control. (D) The tumorsphere formation assay showed that the UTMD transfected CD133+ SMMC-7721 cells formed much smaller and fewer spheroids than cells transduced with Lipofectamine or the control group (magnification, $\mathrm{x} 100$ ). ${ }^{*} \mathrm{P}<0.05$ vs control groups; ${ }^{*} \mathrm{P}<0.05$ vs. $\mathrm{P}+\mathrm{L}$ groups. UTMD, ultrasound-targeted microbubble destruction.

and sphere-forming ability. The UTMD-transfected CD133+ SMMC-7721 cells formed much smaller and fewer spheroids than cells transduced with Lipofectamine or the control group (Fig. 2D). Of note, CD133-transduced spheroids were not passaged from one generation to another, whereas the CD133 ${ }^{+}$ SMMC-7721 cells spheres were passaged, demonstrating their reduced self-renewal ability in vitro. These data suggested that the downregulation of CD133 inhibited self-renewal and the sphere-forming ability of LCSCs and UTMD exerted a more significant knockdown effect on gene transfection in CD133 ${ }^{+}$ SMMC-7721 cells than the Lipofectamine-transfected group.

Downregulation of CD133 suppresses proliferation, colony formation ability and promotes apoptosis in $\mathrm{CD}_{133^{+}}$ SMMC-7721 cells. To detect the biological characteristics of CD133-downregulated LCSCs, colony-forming and proliferation assays were performed. CCK-8 assays showed that CD133+ SMMC-7721 cells transfected with shCD133 
A

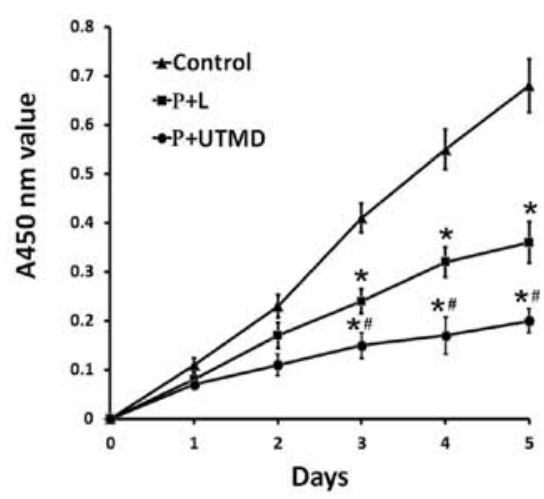

B
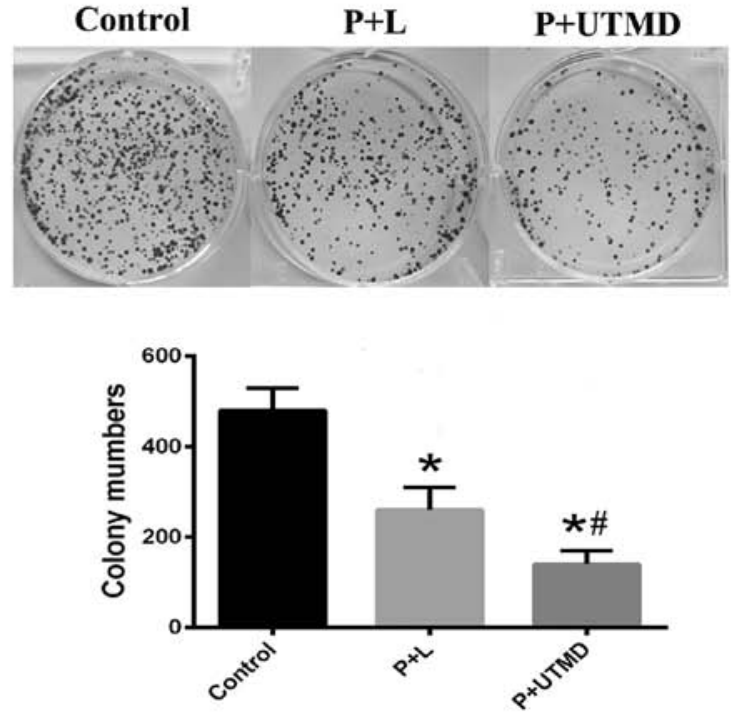

C
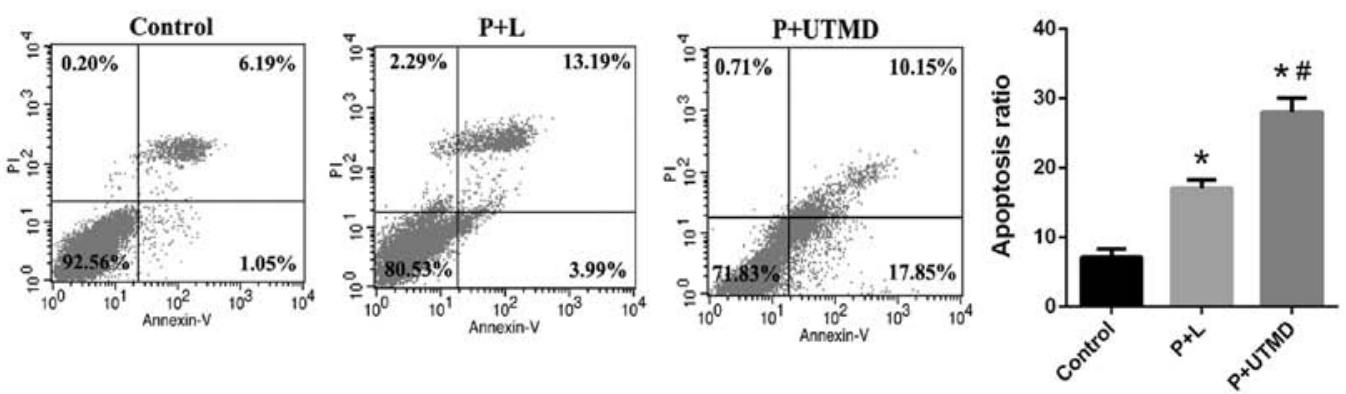

D
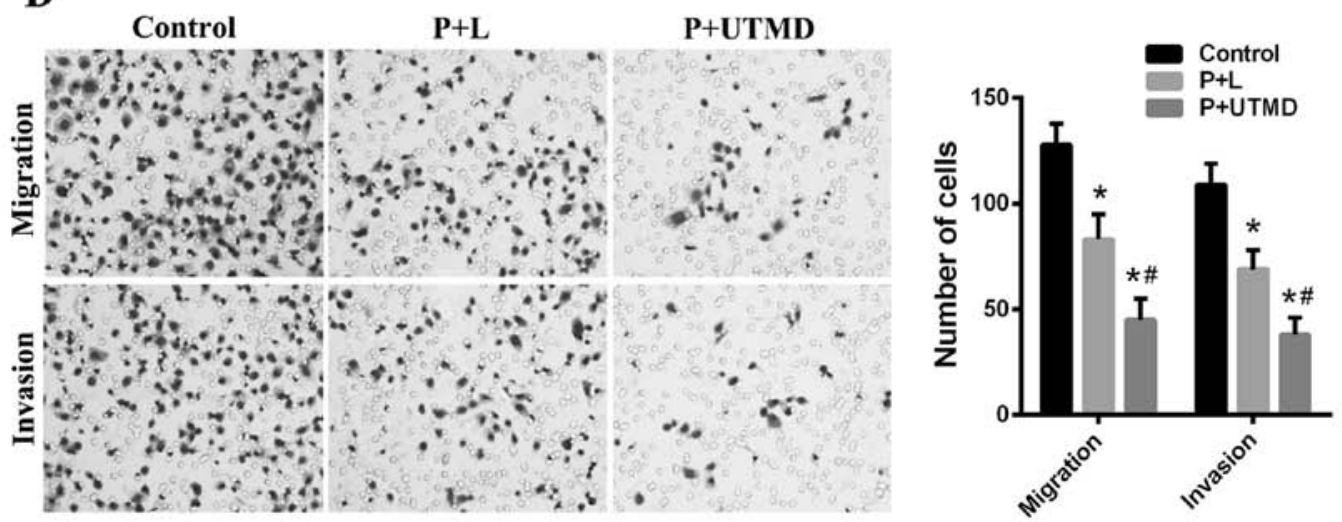

Figure 3. Downregulation of CD133 suppresses proliferation, clonogenicity, migration and invasion and promotes apoptosis in CD133+ SMMC-7721 cells (A) The proliferation rate was decreased in the P+UTMD group, compared to P+L or the control group, based on the CCK-8 assays. (B) In colony-forming assays, the P+UTMD group presented a marked decrease in the number and size of colonies. Upper, representative images; lower, colony numbers in three independent experiments. (C) The percentage of total apoptotic cells (early + late apoptotic) in the P+UTMD group was significantly increased compared with the $\mathrm{P}+\mathrm{L}$ or the control groups based on flow cytometric analysis. (D) Representative images (left, magnification, $\mathrm{x} 200$ ) and the quantifications (right) of cell migration and invasion assays showing that the downregulation of CD133 mediated by UTMD attenuated the migration and invasion of LCSCs. ${ }^{*}<0.05$ vs. control groups; ${ }^{\text {P }}<0.05$ vs. P+L groups. UTMD, ultrasound-targeted microbubble destruction; CCK-8, Cell Counting Kit-8; LCSCs, liver cancer stem cells.

mediated by UTMD (P+UTMD group) had a significantly reduced proliferation rate, compared to the cells transfected with shCD133 mediated by Lipofectamine $(\mathrm{P}+\mathrm{L})$ and the control groups $(\mathrm{P}<0.05$; Fig. $3 \mathrm{~A})$. In the colony-forming assays, the $\mathrm{P}+\mathrm{UTMD}$ group presented a significant decrease in the number and size of colonies $(\mathrm{P}<0.05$; Fig. $3 \mathrm{~B})$. We detected cell apoptosis using Annexin V/PI staining. LCSCs transfected with shCD133 mediated by UTMD significantly increased the percentage of total apoptotic cells (early + late apoptotic) (Fig. 3C). These results suggested that the downregulation of CD133 mediated by UTMD resulted in a reduction of proliferation and cell viability in CD133 ${ }^{+}$LCSCs.

Downregulation of CD133 inhibits invasion and migration in $C D 133^{+}$SMMC-7721 cells. To assess whether the downregulation of CD133 affected the malignant properties of CD133 ${ }^{+}$ LCSCs, we performed the Transwell migration and Boyden chamber assays. As shown in Fig. 3D, the number of LCSCs in the CD133 transfected by UTMD group, migrating or invading through the Boyden chamber pores decreased significantly 

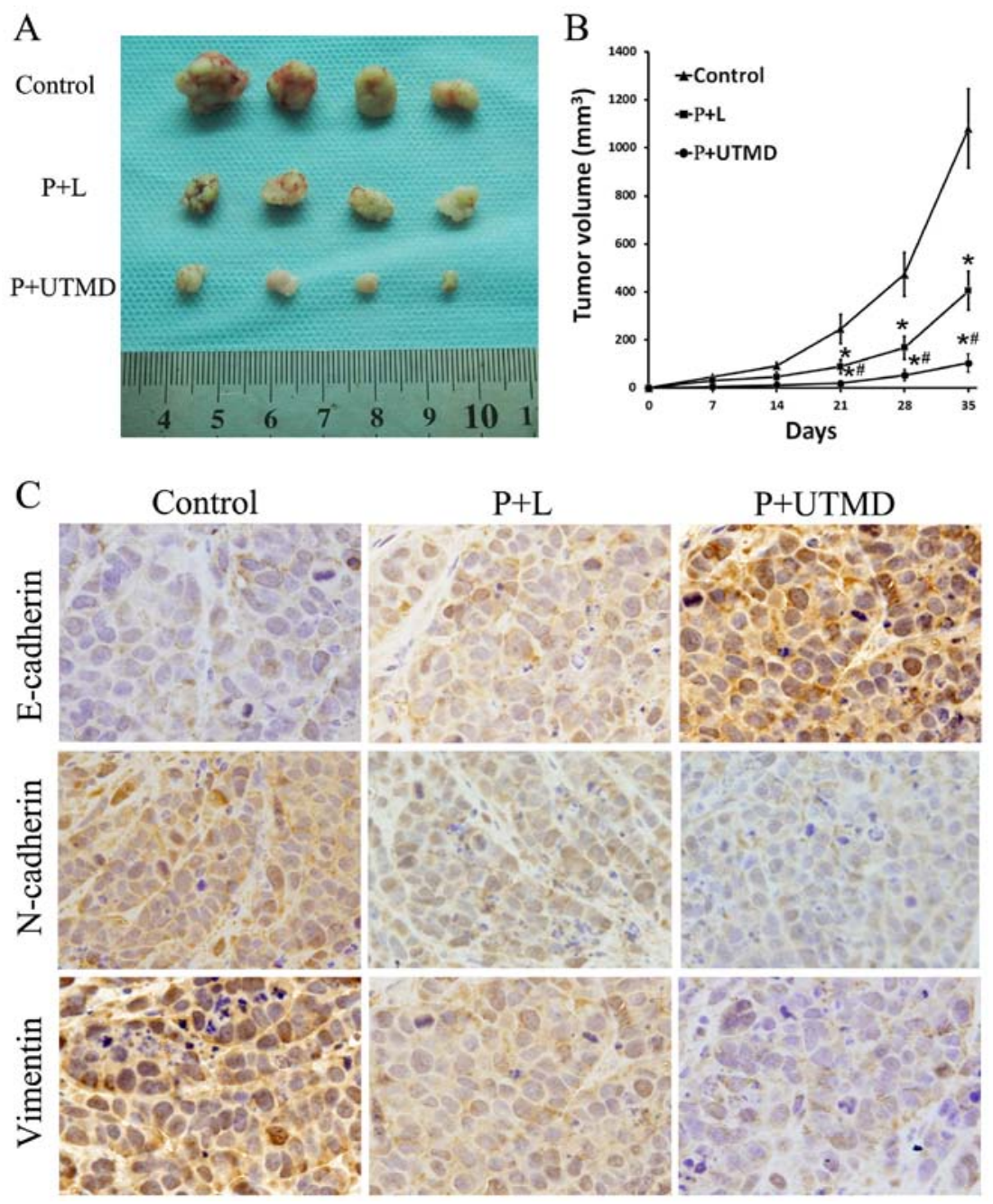

Figure 4. Downregulation of CD133 attenuates tumorigenicity in vivo. (A) Representative xenograft tumors. (B) Tumor volumes were monitored over time. $\left({ }^{*} \mathrm{P}<0.05\right.$ vs. control groups; ${ }^{~} \mathrm{P}<0.05$ vs. $\mathrm{P}+\mathrm{L}$ groups). (C) Representative expression levels of E-cadherin, $\mathrm{N}$-cadherin, and vimentin in the tumor nodules of the nude mice by immunohistochemistry. Original magnification, x400. UTMD, ultrasound-targeted microbubble destruction.

compared with the $\mathrm{P}+\mathrm{L}$ and control groups $(\mathrm{P}<0.05)$. These results suggested that the downregulation of $\mathrm{CD} 133$ inhibited invasion and migration in CD133 ${ }^{+}$LCSCs and that inhibition of CD133 by UTMD may be an attractive method to regulate malignant properties of LCSCs.

Downregulation of CD133 attenuates tumorigenicity in vivo. Based on the observed decreases in proliferative, invasive and migratory behaviors in LCSCs transfected with shCD133 mediated by UTMD, we then determined the effect of CD133 downregulation on the tumorigenic ability of LCSCs using a nude mouse xenograft model. CD133 ${ }^{+}$SMMC-7721 cells were collected by FACS, transfected with shCD133 mediated by UTMD or Lipofectamine for $48 \mathrm{~h}$ and injected into the flank regions of nude mice. During a 5-week follow-up period, it was observed that the tumor volumes varied between groups. CD133-downregulated groups generated small tumors in nude mice in contrast to the large tumors generated in the $\mathrm{CD} 133^{+}$ LCSCs groups. The weights and volumes of tumors in the $\mathrm{P}+\mathrm{UTMD}$ group were significantly reduced compared to those of the $\mathrm{P}+\mathrm{L}$ and control groups $(\mathrm{P}<0.05$; Fig. $4 \mathrm{~A}$ and $\mathrm{B})$. The results suggested the downregulation of CD133 attenuated the ability CD133+ LCSCs to initiate tumors in vivo. Thus,
CD133 served as a target for modulating liver cancer. CD133 downregulated by the UTMD technology exerted a significant effect on the inhibition of tumor growth.

Downregulation of CD133 reverses EMT in the CD133+ SMMC-7721 cells. EMT has been recognized as a de-differentiation program attributed to the generation of CSCs, which is also important in the maintenance of CSCs properties. Based on the earlier results, we examined whether CD133 influenced the expression of several EMT-associated genes. The expression levels of EMT markers were detected by RT-qPCR and western blotting. The results revealed that E-cadherin was enhanced, whereas $\mathrm{N}$-cadherin and vimentin were decreased in CD133-downregulated LCSCs when compared with the CD133 ${ }^{+}$SMMC-7721 cells. The expression of the N-cadherin and vimentin protein in the UTMD-transfected group was lower than that of the Lipofectamine-transfected and control groups, while the expression level of E-cadherin in the UTMD-transfected group was significantly increased $(\mathrm{P}<0.05$; Fig. 5A-C). To investigate the potential mechanisms of CD133 and EMT progress, xenograft tissue samples were analyzed by immunohistochemical staining for the EMT-related proteins E-cadherin, N-cadherin and vimentin. As is evident in Fig. 4C, 

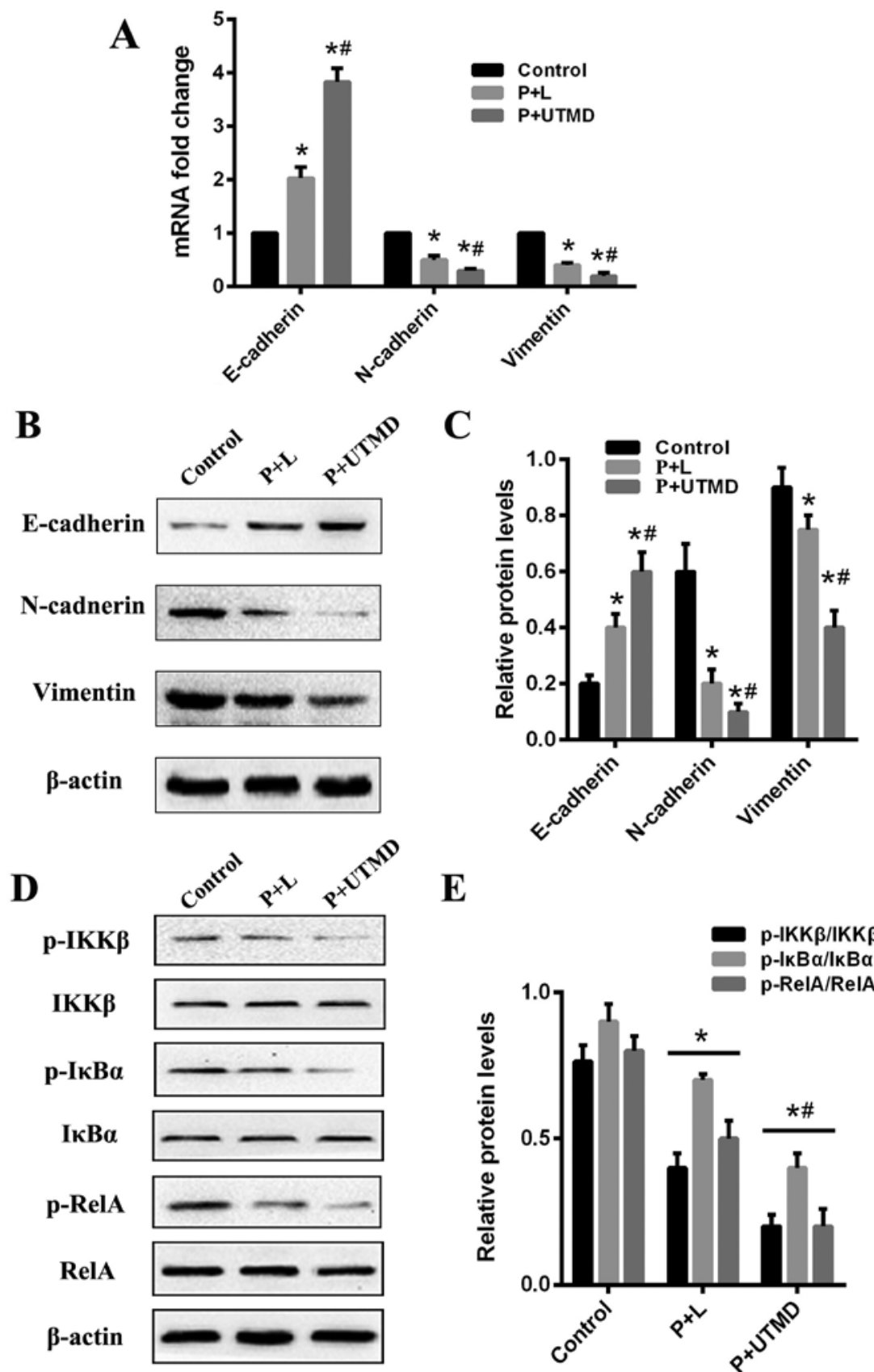

$\mathbf{E}$

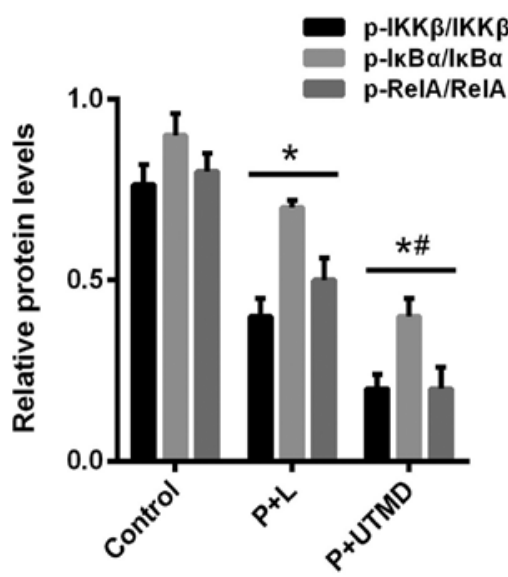

Figure 5. Downregulation of CD133 reversed EMT by suppressing the NF-кB pathway. (A) The mRNA expression levels of the EMT markers (E-cadherin, $\mathrm{N}$-cadherin and vimentin) in the three groups of cells were measured by RT-qPCR. (B and C) The protein levels of the EMT markers in the three groups of cells were evaluated by western blotting. (D and E) Western blot analysis and the relative protein levels of p-IKK $\beta$, p-IкB $\alpha$ and p-RelA in LCSCs. $\beta$-actin served as

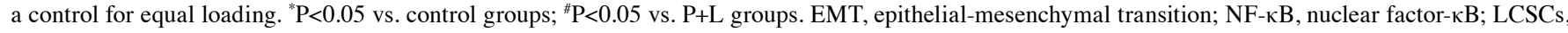
liver cancer stem cells; UTMD, ultrasound-targeted microbubble destruction.

the expression levels of E-cadherin were increased in the UTMD group compared to the $\mathrm{P}+\mathrm{L}$ and control groups $(\mathrm{P}<0.05)$. By contrast, $\mathrm{N}$-cadherin and vimentin exhibited reduced expression levels in the $\mathrm{P}+\mathrm{UTMD}$ group with respect to the $\mathrm{P}+\mathrm{L}$ and control groups, which was consistent with our earlier in vitro studies. These results demonstrated that the EMT process may be reversed by the downregulation of CD133 which contributed to the decreased invasiveness of LCSCs.

Reversal of EMT mediated by CD133 downregulation may be achieved by suppressing the $N F-\kappa B$ pathway. To elucidate the underlying mechanism of CD133 regulation of EMT traits, we focused on the NF- $\kappa \mathrm{B}$ pathway since it has been reported to regulate EMT and the development of CSCs. We identified that the expression of CD133 regulated tumor-initiating properties and the EMT traits. To investigate whether CD133-induced EMT is associated with the $\mathrm{NF}-\kappa \mathrm{B}$ signaling pathway in $\mathrm{CD}_{133}{ }^{+} \mathrm{LCSC}$, we examined the expression of I $\mathrm{B}$ kinase $\beta$ (IKK $\beta)$, inhibitor nuclear factor- $\kappa \mathrm{B} \alpha(\mathrm{I} \kappa \mathrm{B} \alpha)$, and nuclear factor $\kappa \mathrm{B}(\mathrm{NF}-\kappa \mathrm{B})$ RelA using western blotting. By shRNA-mediated knockdown of CD133, a decreased expression of the classical $\mathrm{NF}-\kappa \mathrm{B}$ signaling pathway (IKK $\beta-\mathrm{I} \kappa \mathrm{B} \alpha-\mathrm{RelA}$ ) was observed in the UTMD- and Lipofectamine-transfected groups, as compared with the control group (Fig. 5D and E). This down- 
regulation of the $\mathrm{NF}-\kappa \mathrm{B}$ signaling pathway further correlated with our previous migration analysis and reversal of the EMT phenotype in the LCSCs. Taken together, the results indicated that the NF- $\kappa \mathrm{B}$ pathway mediates the role of CD133 in regulating EMT phenotype.

\section{Discussion}

Cancer stem cells (CSCs) are defined as a small minority of heterogeneous tumorigenic cells that have the ability for self-renewal, differentiation and the potential to proliferate extensively (3). CSCs are considered to be integral to the initiation, progression and metastasis of human types of cancer (4). CD133 has recently been identified as one of the most important CSCs marker for many types of tumor, including liver cancer (5-7). CD133 ${ }^{+}$LCSCs bear features that include the ability to self-renew, differentiate, initiate tumors in vivo and resist standard chemotherapy (8). The underlying biological functions of CD133 remain to be elucidated. Although various studies have shown that CD133 is involved in metastasis, the expression of CD133 has also been identified as an important risk factor of advanced disease stage and worst overall survival in HCC $(9,10)$. In order to determine the molecular mechanisms of CD133 and identify new effective therapeutic approaches for liver cancer, we sorted $\mathrm{CD} 133^{+}$cells from the SMMC-7721 cell line and subsequently inhibited CD133 expression in these cells using a UTMD technique. We demonstrated that the downregulation of CD133 mediated by UTMD significantly attenuated self-renewal, cell proliferation, repressed cell invasion and migration, and inhibited the tumorigenicity in vivo, which was consistent with previous findings for LCSCs $(8,20)$. CD133 expression was also found to be associated with several stem cell-associated genes, including CD44, CD90, Oct4 and Sox2. Thus, we confirm that CD133 plays a function rule in regulating proliferative, migratory behaviors and tumorigenesis in LCSCs.

EMT is a key developmental program that generates cells with properties of stem cells and contributes to tumor initiation, invasion and metastatic spread $(11,12)$. Previous studies have attempted to determine the functional relevance of CD133 and the EMT process in several types of cancer (21-24). The results identified indicated that CD133 may be a critical mediator facilitating migration and invasion through the EMT progress. In the present study, we showed that CD133 regulated the invasive ability and properties of LCSCs. We also examined whether CD133 modulated the EMT pathway in LCSCs. Western blotting and immunohistochemical stainings showed that an epithelial-like protein expression pattern (E-cadherin) was enhanced but a mesenchymal-like protein expression pattern ( $\mathrm{N}$-cadherin and vimentin) was decreased in CD133-downregulated LCSCs. These results indicate that the downregulation of CD133 reversed the EMT process and that EMT mediated by CD133 may be a mechanism for the regulation LCSC initiation, invasion and migration.

$\mathrm{NF}-\kappa \mathrm{B}$ is thought to initiate and accelerate tumorigenesis $(25,26)$. It has been shown that $\mathrm{NF}-\kappa \mathrm{B}$ activation, through regulation of the expression of several transcription factors, promotes the EMT program in cancer cells (27). It has also been suggested that EMT and the process of invasion are regulated by $\mathrm{NF}-\kappa \mathrm{B}$-mediated signaling in $\mathrm{CD} 133^{+}$cancer cells. Nomura et al reported that NF- $\kappa \mathrm{B}$ activation by CD133 surface expression increased the metastatic potential and induced EMT in pancreatic cancer (28). Since NF- $\kappa$ B pathways have been associated with EMT and the development of CSCs, we hypothesized that the reversal of LCSC EMT by downregulated CD133 may be mediated through the NF- $\kappa \mathrm{B}$ signaling pathway. Notably, in the CD133 downregulated LCSCs, E-cadherin levels were increased, whereas N-cadherin and vimentin levels were decreased. Furthermore, a decreased expression of IKK $\beta$-I $\kappa \mathrm{B} \alpha$-RelA phosphorylation was observed, indicating that the downregulation of CD133 reversed the progress of EMT and inhibited the classical NF- $\kappa \mathrm{B}$ signaling pathway in the malignant transformation of LCSCs. These results suggest that $\mathrm{NF}-\kappa \mathrm{B}$ pathway may partially, mediate the role of CD133 in regulating EMT phenotype.

UTMD, as a promising method for gene and drug delivery, may be combined with RNAi technique successfully (16). The UTMD system is the combination of ultrasound and microbubbles, which is safer and more effective compared with other methods. SonoVue is an aqueous suspension of stabilized sulfur hexafluoride microbubbles, which is widely used in the clinic. Ultrasound microbubble-mediated destruction may increase cell membrane permeability and synergistically promote gene delivery. UTMD has previously been used to efficiently deliver plasmid DNA to a variety of cancer cells, including glioma (17), yolk sac carcinoma (18), human cervical cancer (19), bone marrow stromal (29) and HCC cells (30). The aim of the present study was to evaluate the possibility of shRNA vector transfection mediated by UTMD in LCSCs. We also addressed the issue of whether the UTMD-based shRNA delivery system facilitated gene delivery in LCSCs. Under the selected condition, it was found that the transfection mediated by UTMD was higher than that of the Lipofectamine group and showed a significantly decreased expression of CD133, which was in agreement with the results of RT-qPCR and western blot analysis. In addition, the invasiveness and tumorigenicity of LCSCs were significantly decreased by the UTMD transfection of CD133. The present study demonstrates that UTMD is a safe and efficient technique for gene delivery to LCSCs.

In conclusion, the present findings have shown that CD133 expression regulated EMT and stem cell properties in LCSCs in vitro and in vivo. Downregulation of CD133 reduced tumor-initiating activities and inhibited invasion and migratory ability of LCSCs. Notably, the downregulation of CD133 led to a reduced mesenchymal marker (N-cadherin and vimentin) but induced epithelial markers (E-cadherin) in LCSCs, and supported a potential connection between CD133 and EMT transformation. Furthermore, downregulation of the classical NF- $\mathrm{B}$ pathway (IKK $\beta$-I $\kappa \mathrm{B} \alpha-\mathrm{Rel} A$ phosphorylation) was observed in the CD133-downregulated LCSCs, indicating the reversal of EMT and impaired migratory potential of $\mathrm{CD}_{133^{+}}$LCSCs may be in part mediated by suppressing the $\mathrm{NF}-\kappa \mathrm{B}$ signaling pathway. UTMD effectively transfered shCD133 into CD133 ${ }^{+}$LCSCs and led to the inhibition of CD133 expression and the properties of LCSCs. Thus, UTMD may be a powerful and effective tool for the transfection of specific genes and functional analysis of genes, which may be explored as a useful therapeutic option for liver cancer therapy. 


\section{Acknowledgements}

This study was supported by the Research Projects of the Chongqing Municipal Health Bureau (grant nos. 2013-2-080 and 2013-1-022).

\section{References}

1. Forner A, Llovet JM and Bruix J: Hepatocellular carcinoma. Lancet 379: 1245-1255, 2012.

2. Tanaka S and Arii S: Molecularly targeted therapy for hepatocellular carcinoma. Cancer Sci 100: 1-8, 2009.

3. Dalerba P, Cho RW and Clarke MF: Cancer stem cells: Models and concepts. Annu Rev Med 58: 267-284, 2007.

4. Jordan CT, Guzman ML and Noble M: Cancer stem cells. N Engl J Med 355: 1253-1261, 2006.

5. Suetsugu A, Nagaki M, Aoki H, Motohashi T, Kunisada T and Moriwaki $\mathrm{H}$ : Characterization of $\mathrm{CD}_{133^{+}}$hepatocellular carcinoma cells as cancer stem/progenitor cells. Biochem Biophys Res Commun 351: 820-824, 2006.

6. Yin S, Li J, Hu C, Chen X, Yao M, Yan M, Jiang G, Ge C, Xie H, Wan D, et al: CD133 positive hepatocellular carcinoma cells possess high capacity for tumorigenicity. Int J Cancer 120: 1444-1450, 2007.

7. Ma S, Chan KW, Hu L, Lee TK, Wo JY, Ng IO, Zheng BJ and Guan XY: Identification and characterization of tumorigenic liver cancer stem/progenitor cells. Gastroenterology 132: 2542-2556, 2007.

8. Tang KH, Ma S, Lee TK, Chan YP, Kwan PS, Tong CM, Ng IO, Man K, To KF, Lai PB, et al: CD133+ liver tumor-initiating cells promote tumor angiogenesis, growth, and self-renewal through neurotensin/interleukin-8/CXCL1 signaling. Hepatology 55: 807-820, 2012.

9. Yang XR, Xu Y, Yu B, Zhou J, Qiu SJ, Shi GM, Zhang BH, Wu WZ, Shi YH, Wu B, et al: High expression levels of putative hepatic stem/progenitor cell biomarkers related to tumour angiogenesis and poor prognosis of hepatocellular carcinoma. Gut 59: 953-962, 2010

10. Song W, Li H, Tao K, Li R, Song Z, Zhao Q, Zhang F and Dou K: Expression and clinical significance of the stem cell marker CD133 in hepatocellular carcinoma. Int J Clin Pract 62 1212-1218, 2008.

11. Thiery JP, Acloque H, Huang RY and Nieto MA: Epithelialmesenchymal transitions in development and disease. Cell 139: 871-890, 2009.

12. Mani SA, Guo W, Liao MJ, Eaton EN, Ayyanan A, Zhou AY, Brooks M, Reinhard F, Zhang CC, Shipitsin M, et al: The epithelial-mesenchymal transition generates cells with properties of stem cells. Cell 133: 704-715, 2008.

13. Polyak K and Weinberg RA: Transitions between epithelial and mesenchymal states: Acquisition of malignant and stem cell traits. Nat Rev Cancer 9: 265-273, 2009.

14. Song R, Song H, Liang Y, Yin D, Zhang H, Zheng T, Wang J, Lu Z, Song X, Pei T, et al: Reciprocal activation between ATPase inhibitory factor 1 and NF- $\mathrm{BB}$ drives hepatocellular carcinoma angiogenesis and metastasis. Hepatology 60: 1659-1673, 2014.

15. Jiang R, Li Y, Xu Y, Zhou Y, Pang Y, Shen L, Zhao Y, Zhang J, Zhou J, Wang X, et al: EMT and CSC-like properties mediated by the IKK $\beta / \mathrm{I} \kappa \mathrm{B} \alpha /$ RelA signal pathway via the transcriptional regulator, Snail, are involved in the arsenite-induced neoplastic transformation of human keratinocytes. Arch Toxicol 87: 991-1000, 2013.
16. Li HL, Zheng XZ, Wang HP, Li F, Wu Y and Du LF: Ultrasoundtargeted microbubble destruction enhances AAV-mediated gene transfection in human RPE cells in vitro and rat retina in vivo. Gene Ther 16: 1146-1153, 2009.

17. Wang JF, Wu CJ, Zhang CM, Qiu QY and Zheng M: Ultrasoundmediated microbubble destruction facilitates gene transfection in rat C6 glioma cells. Mol Biol Rep 36: 1263-1267, 2009.

18. He Y, Bi Y, Hua Y, Liu D, Wen S, Wang Q, Li M, Zhu J, Lin T, He D, et al: Ultrasound microbubble-mediated delivery of the siRNAs targeting MDR1 reduces drug resistance of yolk sac carcinoma L2 cells. J Exp Clin Cancer Res 30: 104, 2011.

19. Hao Y, Guo L, Abudula A, Saidoula W and Guo X: Proliferation inhibition and apoptosis enhancement of human cervical cancer cells by ultrasound-targeted microbubble destruction delivered double suicide genes. Int J Clin Exp Med 7: 5330-5335, 2014.

20. Lan X, Wu YZ, Wang Y, Wu FR, Zang CB, Tang C, Cao S and Li SL: CD133 silencing inhibits stemness properties and enhances chemoradiosensitivity in CD133-positive liver cancer stem cells. Int J Mol Med 31: 315-324, 2013.

21. Na DC, Lee JE, Yoo JE, Oh B-K, Choi GH and Park YN: Invasion and EMT-associated genes are up-regulated in B viral hepatocellular carcinoma with high expression of CD133-human and cell culture study. Exp Mol Pathol 90: 66-73, 2011.

22. Ding Q, Miyazaki Y, Tsukasa K, Matsubara S, Yoshimitsu M and Takao S: CD133 facilitates epithelial-mesenchymal transition through interaction with the ERK pathway in pancreatic cancer metastasis. Mol Cancer 13: 15, 2014.

23. Zarkoob H, Taube JH, Singh SK, Mani SA and Kohandel M Investigating the link between molecular subtypes of glioblastoma, epithelial-mesenchymal transition, and CD133 cell surface protein. PLoS One 8: e64169, 2013.

24. Chen YS, Wu MJ, Huang CY, Lin SC, Chuang TH, Yu CC and Lo JF: CD133/Src axis mediates tumor initiating property and epithelial-mesenchymal transition of head and neck cancer. PLoS One 6: e28053, 2011.

25. Yang Y, Li Y, Wang K, Wang Y, Yin W and Li L: P38/NF-кB/ snail pathway is involved in caffeic acid-induced inhibition of cancer stem cells-like properties and migratory capacity in malignant human keratinocyte. PLoS One 8: e58915, 2013.

26. Kumar M, Allison DF, Baranova NN, Wamsley JJ, Katz AJ, Bekiranov S, Jones DR and Mayo MW: NF- $\kappa \mathrm{B}$ regulates mesenchymal transition for the induction of non-small cell lung cancer initiating cells. PLoS One 8: e68597, 2013.

27. Wamsley JJ, Kumar M, Allison DF, Clift SH, Holzknecht CM, Szymura SJ, Hoang SA, Xu X, Moskaluk CA, Jones DR, et al: Activin upregulation by NF- $\kappa \mathrm{B}$ is required to maintain mesenchymal features of cancer stem-like cells in non-small cell lung cancer. Cancer Res 75: 426-435, 2015.

28. Nomura A, Banerjee S, Chugh R, Dudeja V, Yamamoto M, Vickers SM and Saluja AK: CD133 initiates tumors, induces epithelial-mesenchymal transition and increases metastasis in pancreatic cancer. Oncotarget 6: 8313-8322, 2015.

29. Wang G, Zhuo Z, Zhang Q, Xu Y, Wu S, Li L, Xia H and Gao Y: Transfection of CXCR-4 using microbubble-mediated ultrasound irradiation and liposomes improves the migratory ability of bone marrow stromal cells. Curr Gene Ther 15: 21-31, 2015.

30. Yu BF, Wu J, Zhang Y, Sung HW, Xie J and Li RK: Ultrasoundtargeted HSVtk and Timp3 gene delivery for synergistically enhanced antitumor effects in hepatoma. Cancer Gene Ther 20: 290-297, 2013. 